\title{
Perception of temporal order during the attentional blink: Using stimulus salience to modulate prior entry
}

\author{
Hayley E. P. Lagroix ${ }^{1}$ - James W. Patten ${ }^{1} \cdot$ Vincent Di Lollo $^{1} \cdot$ Thomas M. Spalek $^{1}$
}

Published online: 16 February 2016

(C) The Psychonomic Society, Inc. 2016

\begin{abstract}
When multiple targets are presented in rapid sequence, observers frequently confuse the order in which they were presented. The probability of order reversals is known to vary throughout the period of the attentional blink $(\mathrm{AB})$, which refers to impairment in the perception of the second of two targets when it is presented within approximately $500 \mathrm{~ms}$ from the first. Our objective was to examine the principle of prior entry (in which perception of temporal order is said to be affected by the relative latency at which each target is processed) as a determinant of the perception of temporal order throughout the $\mathrm{AB}$. In two experiments, three letter targets $(\mathrm{T} 1, \mathrm{~T} 2, \mathrm{~T} 3)$ were inserted in a stream of digit distractors, with $\mathrm{T} 3$ always presented directly after $\mathrm{T} 2$. The T1-T2 lag was varied to assess the perception of T2-T3 temporal order throughout the period of the AB. Processing latency was manipulated by means of salience. In Experiment 1, salience of $\mathrm{T} 2$ and T3 was manipulated exogenously by coloring the salient target red with all other stimuli being green. In Experiment 2, salience was modulated endogenously by manipulating which of the two targets matched the contents of working memory. Consistent with the principle of prior entry, perception of temporal order in both experiments was enhanced throughout the period of the $A B$ when T2 was salient, and impaired when T3 was salient. Simulations based on the Episodic Simultaneous Type, Serial Token (eSTST) model that incorporates prior-entry, matched the empirical results.
\end{abstract}

Hayley E. P. Lagroix

hlagroix@sfu.ca

1 Department of Psychology, Simon Fraser University, 8888 University Drive, Burnaby, BC, Canada V5A 1S6
Keywords Theoretical and Computational Models . Attentional blink $\cdot$ Selective attention

When two stimuli are presented in rapid sequence, observers frequently confuse the order in which they were presented. Among the factors thought to determine the perception of temporal order is the relative amount of attention deployed to the stimuli. That is, the perception of temporal order of two sequential stimuli is determined not only by the actual order of presentation but also by the relative amount of attention deployed to the stimuli (Reeves \& Sperling, 1986). Thus, a well-attended temporally-trailing stimulus may be perceived as having occurred earlier than a less-attended stimulus that was actually presented first. This is known as the principle of prior entry (Spence \& Parise, 2010; Titchener, 1908). ${ }^{1}$ There is an abundance of findings that show that prior entry can be elicited by peripheral as well as central cues or exogenous as well as endogenous attention (Shore, Spence \& Klein, 2001).

On the face of it, the role of attention in the perception of temporal order could be examined with a paradigm known as the attentional blink (AB). Originally demonstrated by Raymond, Shapiro, and Arnell (1992), the AB refers to an impairment in identification accuracy for the second of two

\footnotetext{
${ }^{1}$ There is an interesting and enduring discussion as to whether or to what extent prior entry reflects judgment biases or reflects the influence of attention on processing rate (Jaskowski, 1993; Shore, Spence, \& Klein, 2001; Spence, Shore, \& Klein, 2001) and also whether any changes in processing rate involve the attended or the unattended item (Tünnermann, Petersen \& Scharlau 2015; Weiss, Hilkenmeier \& Scharlau 2013). The assumption is made in the present work that prior entry speeds up the processing of the attended item. A detailed discussion of these issues, however, would be beyond the scope of the present work. This is because the principal objective of the present study was not to examine the mechanisms underlying prior entry but to determine whether prior entry affects the perception of temporal order throughout the course of the AB.
} 
targets (T2) when it is presented within about $500 \mathrm{~ms}$ from the first (T1). Conventionally, the two targets are inserted in a stream of distractors presented in rapid serial visual presentation (RSVP). The T2 deficit has been ascribed to an uneven deployment of attention during the period of the $A B$, when attention is deployed to $\mathrm{T} 1$ to the detriment of $\mathrm{T} 2$.

As for the perception of temporal order, there is evidence that, despite instructions to report $\mathrm{T} 1$ and $\mathrm{T} 2$ in the order in which they were presented, observers often report them in the reverse order, with the frequency of reversals being highest when $\mathrm{T} 2$ is presented directly after T1, in the RSVP position known as Lag 1 (Akyürek et al., 2012; Akyürek \& Hommel, 2005; Akyürek, Riddell, Toffanin, \& Hommel, 2007; Chun, 1997; Chun \& Potter, 1995; Hilkenmeier, Scharlau, Weiss, \& Olivers, 2012; Isaak, Shapiro, \& Martin, 1999; Olivers, Hilkenmeier, \& Scharlau, 2011; Spalek, Falcon, \& Di Lollo, 2006). Interpretation of this finding, however, is complicated by a confounding factor. Namely, as the intertarget lag is increased, the temporal separation between the two targets increases correspondingly, making the targets more temporally distinct from one another. The improved perception of temporal order as the inter-target lag is increased would then be attributable to increased ease of temporal discriminability rather than to improved ability to maintain temporal order.

To determine whether the perception of temporal order is impaired during the period of the $\mathrm{AB}$, it is necessary to keep the temporal discriminability of the two targets constant throughout that period. This was done by Spalek, Lagroix, Yanko, and Di Lollo (2012) who used a variant of the AB paradigm in which three letter targets $(\mathrm{T} 1, \mathrm{~T} 2, \mathrm{~T} 3)$ were inserted in an RSVP stream of digit distractors. The temporal lag between T1 and T2 was 100, 200, or $700 \mathrm{~ms}$ (Lags 1, 2, and 7). Critically, T3 was always presented directly after T2. This permitted an assessment of T2-T3 order errors throughout the period of the $\mathrm{AB}$ (i.e., at each T1-T2 lag). The highest frequency of order errors was observed at Lag 2, with Lags 1 and 7 showing considerably fewer reversals.

Spalek et al. (2012) offered a theoretical account of their results in terms of the Episodic Simultaneous Type, Serial Token (eSTST) model of Wyble, Bowman, and Nieuwenstein (2009), which incorporates the principle of prior entry. Other models have been proposed that also utilize prior entry to account for order reversals. Notably, Hilkenmeier, Scharlau, Weiss, and Olivers (2012) proposed a simple computational model similar to Olivers and Meeter's (2008) Boost-and-Bounce theory, to account for order reversals of two sequential targets inserted in an RSVP stream of distractors. Two things should be noted about this model: first, its predictions were examined only for Lag 1; second, the main conclusion was that the tenets of the model were consistent with a range of other theories that postulate transient attention as the mechanism underlying order reversals (Shih, 2008; Olivers \& Meeter, 2008; Wyble et al., 2009).

Any of these models would provide a suitable conceptual framework for the present work, whose main objective was to study the relationship between prior entry and the $\mathrm{AB}$. We opted for the eSTST model for two reasons: 1) it has been implemented not only for Lag 1 but for lags throughout the period of the $A B$, and 2) it has been used successfully to predict the outcomes of other $\mathrm{AB}$ experiments (Spalek et al., 2012; Lagroix, Spalek, Wyble, Jannati, \& Di Lollo, 2012). A brief outline of the eSTST model will show how it accounts for the results of Spalek et al. and will provide the conceptual background for the present work.

At the most basic level, eSTST makes use of a two-stage architecture similar to that of Chun and Potter's (1995) twostage theory and adopts the type-token distinction introduced by Kanwisher (1987). In the first stage (the input layer), abstract identity information about each stimulus (its type) is extracted. For a stimulus to be reported correctly, however, it must gain access to a later encoding stage where its identity information is bound to a token and is thereby encoded into working memory.

For a type representation to be bound to a token, it must be enhanced by a transient attentional mechanism (the blaster) that amplifies the strength of the type signal. This mechanism is triggered upon detection of a target, and its activity lasts for approximately $200 \mathrm{~ms}$. An inhibitory phase then follows, during which attention (i.e., the blaster) is suppressed while the target undergoes the binding process. This T1-triggered suppression of attention extends over several hundred milliseconds and mediates the $\mathrm{AB}$ by preventing the $\mathrm{T} 2$ type from being bound to a token until T1 tokenization is complete. This delay in $\mathrm{T} 2$ processing leaves it vulnerable to decay and interference by trailing items. On the other hand, if T2 is presented during the excitatory period of the blaster's activity triggered by $\mathrm{T} 1$ (i.e., if it is presented within the same attentional episode as T1), its signal will be amplified. This will result in T2 preceding the inhibitory phase, thus avoiding the $\mathrm{AB}$ deficit, a phenomenon known as Lag-1 sparing. This sequence of processing events culminates with the production of tokens in working memory. Each token has its own unique time stamp which determines the order in which it is perceived.

Within eSTST, two main factors are said to govern the perception of temporal order. First, temporal order is determined both by the order in which the targets were presented and by their relative strength. This is because stronger types are tokenized faster thus increasing the probability of gaining earlier access to working memory. Second, the strength of a type is determined by the degree of enhancement received from the blaster, that is, the amount of attention deployed to it. In essence, eSTST handles the perception of temporal order according to the principle of prior entry which states that attention affects the latency of target processing (Maylor \& 
Hockey, 1985; Töllner, Zehetleitner, Gramann, \& Müller, 2011; but see Stelmach \& Herdman, 1991, for a more complex view of the effects of attention on stimulus processing).

On this principle, any manipulation that causes a greater amount of attention to be deployed to the second of two successive targets will (a) increase its signal strength, which will (b) decrease its processing latency, leading to (c) increased probability of order reversals. Spalek et al. (2012) tested this conjecture by manipulating the latency of attention deployment to two successive targets (T2 and T3, as noted above) in two ways. One way was to manipulate the presence/ absence of distractors intervening between $\mathrm{T} 1$ and $\mathrm{T} 2$ in the RSVP stream. The sequence of events is illustrated in Fig. 1. When distractors are present (Fig. 1A), the deployment of

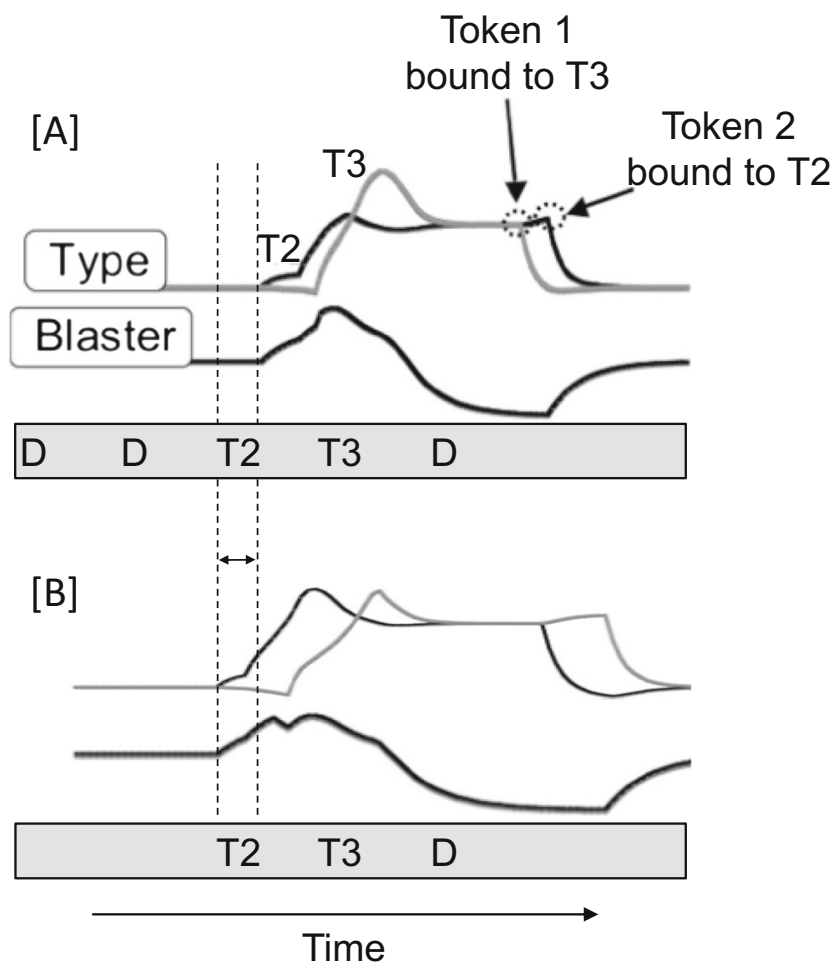

Fig. 1 Schematic depiction of the activation of the blaster and type nodes in the eSTST model in response to two sequential targets (T2, T3). Modified from Figures 12 and 16 of Wyble et al. (2009). (A) Example of a trial on which distractors precede T2. The onset of the blaster is delayed relative to when distractors are absent, resulting in less attention being deployed to $\mathrm{T} 2$ than to $\mathrm{T} 3$, thus causing the strength of the T3-type signal to be enhanced. The enhanced strength of T3 causes it to complete the token-binding process before T2. The outcome of this trial would be that both T2 and T3 are reported but in the wrong order. (B) Example of a trial on which there are no distractors preceding T2. The onset of the blaster is not delayed, resulting in similar amounts of attention being deployed to T2 and T3. The comparable strengths of the targets cause them to be tokenized in the order of their presentation. The outcome of this trial would be that T2 and T3 are reported in the correct order. The delay in the onset of the blaster caused by the presence of distractors preceding $\mathrm{T} 2$ is shown by the time difference between the two vertical segmented lines. $\mathrm{T}=$ target; $\mathrm{D}=$ distractor. attention to T2 (i.e., the onset of the blaster) is delayed relative to when the distractors are absent (Fig. 1B). The delay is shown by the time difference between the two vertical segmented lines in Fig. 1. This delay results in less attention being deployed to T2 than to T3, causing the strength of the T3-type signal to be enhanced, with a corresponding increment in the probability that T3 is tokenized before T2 (Fig. 1A). The upshot is increased frequency of reversals when distractors are present.

The second way in which the latency of attention deployment was manipulated in the Spalek et al. (2012) study was by varying the T1-T2 lag. In terms of eSTST, this manipulation affects the suppression of the blaster. At short lags, the blaster is suppressed while $\mathrm{T} 1$ is being tokenized. This means that the deployment of attention to $\mathrm{T} 2$ is delayed, resulting in less attention being deployed to $\mathrm{T} 2$ relative to $\mathrm{T} 3$. The consequences for the perception of temporal order are the same as for the distractor manipulation described above.

In both these cases (presence/absence of distractors and inter-target lag), the relative strengths of the $\mathrm{T} 2$ and $\mathrm{T} 3$ signals were manipulated indirectly by events that preceded the T2T3 pair. In the present work we manipulated the salience of the stimuli themselves in order to test the principle of prior entry in a different - and more direct-way.

The plan was to manipulate prior entry by varying the relative salience of two sequential targets. We followed Fecteau and Munoz (2006) who defined salience as that property of a stimulus that causes it to be processed faster than a non-salient stimulus. In the present work, we manipulated the relative salience of $\mathrm{T} 2$ and $\mathrm{T} 3$ within the three-target $\mathrm{AB}$ paradigm employed by Spalek et al. (2012). In Experiment 1, we manipulated salience exogenously by varying a physical property of the stimuli (their color). In Experiment 2, we manipulated salience endogenously by exploiting the finding that attention is deployed more rapidly to a stimulus when it matches a representation in working memory (Downing, 2000; Soto, Heinke, Humphreys, \& Blanco, 2005).

As noted above, it has been known for some time that (a) prior entry can be affected by exogenous as well as endogenous attention (Shore, Spence, \& Klein, 2001), (b) that order errors happen in the AB (Spalek et al., 2012), and (c) that salience affects prior entry (Donk \& Soesman, 2011). The novel contribution of the present work is that these factors were examined in combination. Namely, the effects of exogenous and endogenous manipulations of salience on the perception of temporal order were examined throughout the period of the $\mathrm{AB}$.

\section{Experiment 1}

In Experiment 1, we manipulated salience of the target stimuli by means of color. It is known that attention is deployed more 
quickly to (a) red than to green stimuli (Fortier-Gauthier, Dell'Acqua, \& Jolicœur, 2013) and (b) to singleton than to non-singleton items (Theeuwes, 1992). With these considerations in mind, either $\mathrm{T} 2$ or $\mathrm{T} 3$ was made more salient by making it a red singleton amongst green items. In a baseline condition both $\mathrm{T} 2$ and $\mathrm{T} 3$ were green, as the rest of the stream, making them equally salient. This resulted in three conditions (baseline, T2 salient, T3 salient) that were crossed with lag (Lags 1, 2, and 7; Fig. 2). Based on the principle of prior entry, we expected the frequency of order reversals to vary with target salience. Fewer order reversals relative to baseline should be in evidence when T2 is salient and more when T3 is salient.

These predictions were instantiated in the simulation of the eSTST model (Fig. 3A). The simulations were obtained using the same model and parameters as were used by Wyble et al. (2009), with the exception that the effect of salience was simulated by adding a constant of 0.2 to the input-strength value of the salient target. ${ }^{2}$ This value was chosen $a$ priori by the lead author of Wyble et al. (2009) in advance of seeing the results, on the assumption that the representation of the salient target would be moderately stronger at the input level. The results of the simulation were consistent with the principle of prior entry: salience affected the probability of reversals at every lag in the predicted direction.

\section{Method}

Participants Fifty-seven volunteers participated for class credit or payment. All reported normal or corrected-to-normal vision and were naive to the purpose of the experiment.

Apparatus and stimuli The stimuli consisted of sequences of digits (2-9) and three letters (T1, T2, T3; all uppercase letters of the English alphabet, except I, O, Q, and Z) presented in RSVP in the centre of a computer monitor. All digits, as well as T1, were green (CIE x/y values: 287/.627; luminance: $31.0 \mathrm{~cd} / \mathrm{m}^{2}$, as measured by a Minolta CS 100 Chroma Meter). In the baseline condition T2 and T3 also were displayed in green. In the T2-salient condition, T2 was colored red (CIE x/y values: 0.646/0.336; luminance: $32.2 \mathrm{~cd} / \mathrm{m}^{2}$ ) and $\mathrm{T} 3$ green. The reverse was true in the T3-salient condition. All stimuli subtended approximately $0.5^{\circ}$ of visual angle vertically and were presented on a white background (CIE x/y values: 0.308/0.333; luminance: $154 \mathrm{~cd} / \mathrm{m}^{2}$ ). Observers sat in a dimly lit room and viewed the displays binocularly from a distance of approximately $60 \mathrm{~cm}$.

\footnotetext{
${ }^{2}$ As in the study by Spalek et al. (2012), Lag 3 was used instead of Lag 2 in the simulations because, in the present version of eSTST, the transient attentional window that determines the signal strength of items encoded in working memory is probably a little too broad in comparison with the data. This, however, must be regarded as merely a matter of parameter adjustment rather than a fundamental flaw with the model.
}

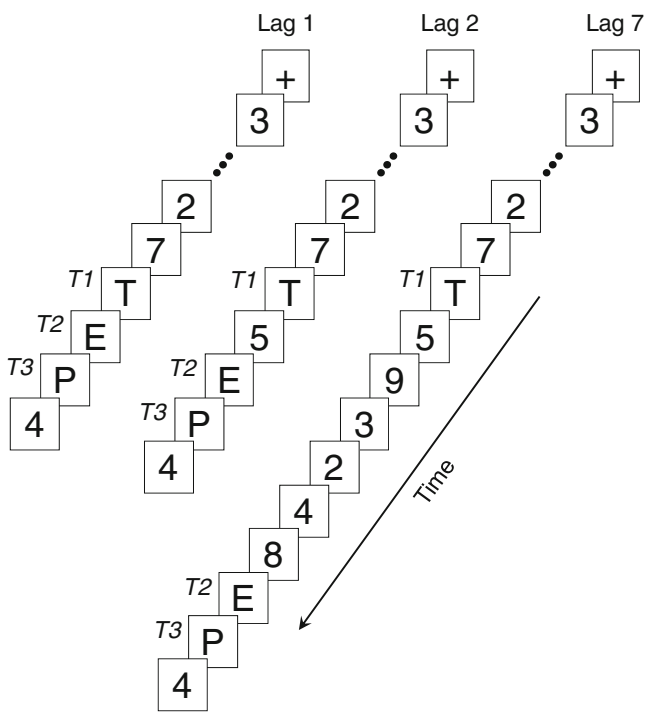

Fig. 2 Schematic representation of the sequence of events within a trial for each lag in Experiment 1. All items were black, except for T2 and T3 one of which was red and the other green on any given trial

Procedure At the beginning of a session, observers were required to read the instructions displayed on the screen, and were invited to ask questions to clarify procedural points. At the beginning of each trial, a small green fixation cross was presented in the center of the screen, indicating the location at which the RSVP stream of digits and letters was about to appear. Observers initiated each trial by pressing the space bar. The RSVP stream of digits and letters was displayed directly afterward. Each item in the RSVP stream remained on the screen for $100 \mathrm{~ms}$, and was replaced immediately by the next item, yielding a presentation rate of $10 \mathrm{items} / \mathrm{s}$.

The RSVP stream contained a variable number of digit distractors and three letter targets, selected randomly, without

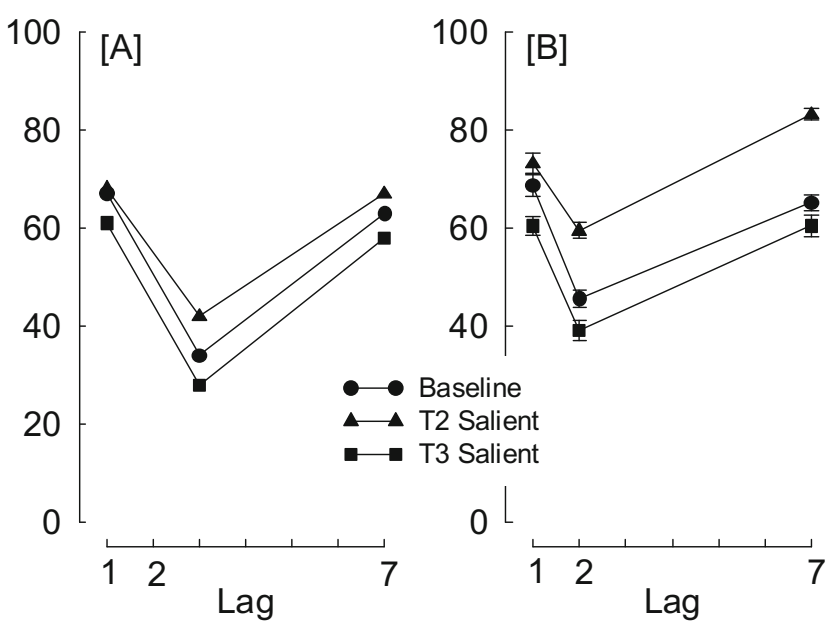

Fig. 3 (A) Simulated results of correct T2-T3 ordered responses for Experiment 1, based on the eSTST model (Wyble et al., 2009). (B) Percentage of correct T2-T3 ordered responses in Experiment 1, only for trials in which all three targets were reported correctly and T1 was reported first. Error bars indicate standard error of the mean 
replacement, from the pool of letters. The number of distractors preceding the first target was determined randomly on each trial and varied between 5 and 10. On any given trial, the distractors were selected randomly, with replacement, from the pool of digits, with the constraint that the selected digit was not one of the two preceding items.

Three targets were inserted in the RSVP stream. T1 was presented directly after the last distractor in the leading stream. T2 was then presented at one of three lags after the onset of T1: 100 (T2 presented directly after T1), 200, or $700 \mathrm{~ms}$. Digit distractors continued to be presented during the two longer intertarget lags. T3 was always presented directly after T2 and was followed by one digit-distractor that acted as a mask (Fig. 2). Observers were required to report the identity of the three targets in the order in which they were presented by pressing the corresponding keys on the keyboard. They were informed that the first letter would always be green, and that either the second or third letter might be red or that both could be green. The combination of the T2-T3 colors was determined randomly on each trial, with the constraint that there were an equal number of trials for each T2-T3 color combination, at each of the three lags.

There were 9 practice trials at the beginning of the session. These were followed by a total of 360 trials, 40 for each of the nine combinations of Salience (Baseline, T2 Salient, T3 Salient) and Lag $(1,2,7)$. The nine combinations were presented randomly intermixed across trials.

\section{Results and discussion}

Identification accuracy Identification accuracy (and associated standard error) for the three targets, averaged over observers, separately for each combination of Salience and Lag, is presented in Table 1 . The values for $\mathrm{T} 2$ and $\mathrm{T} 3$ were conditionalized on the correct report of all leading targets on that trial.

Given that the main focus of the present work was the perception of temporal order as distinct from identification accuracy, no statistical analyses were performed at this point, except to verify that an $\mathrm{AB}$ was obtained. This was done by comparing mean T2 accuracy (given correct report of T1) for Lags 2 and 7 in all three conditions. The analysis revealed significant $\mathrm{AB}$ deficits (T2 accuracy lower at Lag 2 than at Lag 7; Table 1) in all conditions: Baseline: $t(56)=9.39, p<$ 0.001 , T2 Salient: $t(56)=10.51, p<0.001$; T3 Salient: $t(56)=$ $7.97, p<0.001$.

Perception of temporal order The main objective of the present work was to assess the effect of the relative salience of $\mathrm{T} 2$ and $\mathrm{T} 3$ on the perception of T2-T3 temporal order at different T1-T2 lags. For this purpose, it is necessary to consider only trials in which all three targets are reported correctly. The reasoning is as follows: In order to be confident that an
Table 1 Percentage of correct identifications of the three targets (and standard error of the mean)

\begin{tabular}{ccccc}
\hline & & T1 & T2|T1 & T3|T1\&T2 \\
\hline Experiment 1 & & & & \\
Baseline & Lag 1 & $75(1.6)$ & $89(1.1)$ & $72(1.9)$ \\
& Lag 2 & $85(1.4)$ & $57(1.6)$ & $73(1.8)$ \\
& Lag 7 & $86(1.4)$ & $76(1.5)$ & $89(1.2)$ \\
T2 Salient & Lag 1 & $70(1.9)$ & $93(0.8)$ & $70(1.9)$ \\
& Lag 2 & $85(1.3)$ & $71(1.8)$ & $73(2.0)$ \\
& Lag 7 & $86(1.4)$ & $91(0.9)$ & $83(1.4)$ \\
T3 Salient & Lag 1 & $74(1.7)$ & $86(1.2)$ & $81(1.4)$ \\
& Lag 2 & $84(1.5)$ & $53(2.3)$ & $83(1.7)$ \\
Experiment 2 & Lag 7 & $87(1.4)$ & $73(2.0)$ & $94(0.7)$ \\
& & & & \\
& Lag 1 & $79(2.1)$ & $87(1.4)$ & $76(2.1)$ \\
& Lag 2 & $86(1.6)$ & $61(2.2)$ & $72(2.6)$ \\
T2 Salient & Lag 7 & $88(1.4)$ & $81(2.1)$ & $88(1.2)$ \\
& Lag 1 & $76(2.0)$ & $96(0.9)$ & $70(2.5)$ \\
& Lag 2 & $85(1.9)$ & $88(2.3)$ & $66(2.7)$ \\
& Lag 7 & $85(1.7)$ & $94(1.2)$ & $85(1.6)$ \\
& Lag 1 & $76(2.2)$ & $85(1.6)$ & $93(2.1)$ \\
& Lag 2 & $83(1.9)$ & $53(2.8)$ & $93(1.6)$ \\
& Lag 7 & $86(2.0)$ & $74(2.3)$ & $96(1.0)$ \\
\hline
\end{tabular}

$\mathrm{AB}$ occurred on any given trial, $\mathrm{T} 1$ must be reported correctly. Then, to assess the perception of temporal order in the T2-T3 pair, both targets also must be reported correctly. Namely, if only one of them is reported correctly it would be unclear whether or not it had been seen in the correct temporal order. For example, if $\mathrm{T} 2$ is missed, the observers might opt to begin their report with the two items of which they are sure (T1 and $\mathrm{T} 3$, in this example) and then go on to guess the third item. In this example, T3 was perceived in the correct order but was reported in the incorrect order. Thus, the main requirement for scoring temporal order was that $\mathrm{T} 1$ be reported correctly in the first ordinal position and T2 and T3 be reported correctly regardless of order.

Each of the 57 observers contributed 40 trials at each of the nine combinations of Salience (Baseline, T2 Salient, T3 Salient) and Lag $(1,2,7)$, for a total of 2,280 trials per cell. The number of trials on which all three targets were reported correctly with $\mathrm{T} 1$ in the correct position was 750,721 , and 1309 for Lags 1, 2, and 7, respectively, in the Baseline condition, 627, 905, and 1471 for Lags 1, 2, and 7, respectively, in the T2 Salient condition, and 780, 757, and 1340 in the T3 Salient condition. The functions in Fig. 3B illustrate the percentage of trials on which $\mathrm{T} 2$ and $\mathrm{T} 3$ were reported in the correct sequence, given correct identification of all three targets, separately for each condition, across lags. A 3 (Salience: Baseline, T2 Salient, T3 Salient) $\times 3$ (Lag: 1, 2, 7) withinsubjects analysis of variance (ANOVA) performed on the data 
in Fig. 3B yielded significant effects of Salience, $\mathrm{F}(2,112)=$ 71.37, $p<0.001, \mathrm{MSE}=213.44, \eta_{\mathrm{p}}{ }^{2}=0.560$, and Lag, $\mathrm{F}(2$, $112)=99.66, p<0.001, \mathrm{MSE}=241.00, \eta_{\mathrm{p}}{ }^{2}=0.640$. The interaction effect was also significant, $\mathrm{F}(4,224)=5.08, p=$ $0.001, \mathrm{MSE}=147.54, \eta_{\mathrm{p}}{ }^{2}=0.083$.

To ascertain whether each of the two salience manipulations (T2 or T3 salient) differed significantly from baseline, we performed two additional planned comparisons. The T3salient versus Baseline ANOVA revealed significant effects of Salience, $\mathrm{F}(1,56)=24.35, p<0.001, \mathrm{MSE}=147.95, \eta_{\mathrm{p}}{ }^{2}=$ 0.303, and Lag, $\mathrm{F}(2,112)=64.62, p<0.001, \mathrm{MSE}=269.06$, $\eta_{\mathrm{p}}{ }^{2}=0.675$. The interaction effect was not significant $(\mathrm{F}<1)$. The T2-salient vs. Baseline ANOVA revealed significant effects of Salience, $\mathrm{F}(1,56)=56.00, p<0.001, \mathrm{MSE}=223.75$, $\eta_{\mathrm{p}}{ }^{2}=0.500$, and Lag, $\mathrm{F}(2,112)=84.14, p<0.001, \mathrm{MSE}=$ $183.98, \eta_{\mathrm{p}}{ }^{2}=0.600$. The interaction effect was also significant, $\mathrm{F}(2,112)=8.67, p<0.001, \mathrm{MSE}=158.74, \eta_{\mathrm{p}}{ }^{2}=0.134$. We pursued this significant interaction by performing individual $t$ tests between the mean for the Baseline condition and that for the T2-Salient condition, separately for each lag. The only comparison that failed to reach significance was at Lag 1, $t(56)=1.39, p=0.171$ (all other $p s<0.001$ ). Two things should be noted in this respect. First, the difference between the T2-salient and the Baseline condition was numerically in the expected direction. Second, the difference between the T2salient and the T3-salient conditions at Lag 1 did reach statistical significance $(t(56)=4.65, p<0.001)$. These observations are consistent with the idea that the salience manipulation affected the perception of temporal order throughout the period of the AB.

These statistical analyses confirm the graphical evidence in Fig. 3B that, relative to baseline, the perception of temporal order was more accurate when T2 was salient and less accurate when T3 was salient. This pattern of results is consistent with the principle of prior entry which stipulates that perception of the temporal order of two targets is determined by their relative processing speed (here influenced by salience) as well as by the actual order of presentation. In the present experiment, salience and order of presentation worked in conjunction when T2 was salient (enhancing the perception of temporal order) but in opposition when T3 was salient (impairing the perception of temporal order).

It is clear from a comparison of Fig. $3 \mathrm{~A}$ and $\mathrm{B}$ that the effect of salience on the perception of temporal order was predicted accurately by the eSTST model. Remarkably, the simulated results provided an excellent match for the empirical results not only by showing that the three conditions differed from one another at each lag, but also by showing that the T2-Salient condition did not differ statistically from the Baseline condition at Lag 1. The simulated score for the T2-Salient condition at Lag 1 was only $1 \%$ higher than the corresponding score in the Baseline condition.

\section{Experiment 2}

As noted above, the eSTST model is an instance of a broader class of models based on the principle of prior entry (Reeves \& Sperling, 1986). It can be inferred from that principle that salience should have similar effects on the perception of temporal order, whether it is manipulated exogenously or endogenously. The important consideration is that salience affects the latency of processing-and hence the perception of temporal order-regardless of the way in which it is manipulated. The present experiment was a test of this hypothesis.

Instead of manipulating salience exogenously, as in Experiment 1, in the present experiment we manipulated salience endogenously by exploiting the finding that attention is deployed more rapidly to a stimulus when it matches a representation in working memory (Downing, 2000; Soto, Heinke, Humphreys, \& Blanco, 2005).

Accordingly, in the present experiment, all stimuli were presented in black, but T2-T3 salience was manipulated by presenting either the T2 or the T3 letter prior to the RSVP stream, as the fixation item instead of the cross used in Experiment 1. Participants were informed that this letter would match either the second or the third target letter in that trial. With these exceptions, all stimuli and procedures were the same as in Experiment 1.

Predictions stemming from the general principle of prior entry can be instantiated in the eSTST model. The present endogenous manipulation of salience can be modeled in eSTST by reducing the tokenization threshold of the salient target. In practice, this means that the salient target is tokenized more rapidly, thus increasing the probability of it being perceived as temporally leading. In the present simulation, illustrated in Fig. 4A, the tokenization threshold of the salient target was set at $70 \%$ of its nonsalient value. As in Experiment 1, this value was chosen a priori by the lead author of Wyble et al. (2009) prior to seeing the results. This was done under the assumption that, while observers knew the identity of the salient target ahead of time, they still needed to encode other information, such as its temporal and spatial characteristics. This would have caused only a modest reduction in the amount of information in need of consolidation into memory.

\section{Method}

Participants Thirty-five volunteers participated for class credit or payment. They were drawn from the same population as in Experiment 1.

Apparatus and stimuli The apparatus and stimuli were the same as in Experiment 1, with the exception that all stimuli were presented in black. 


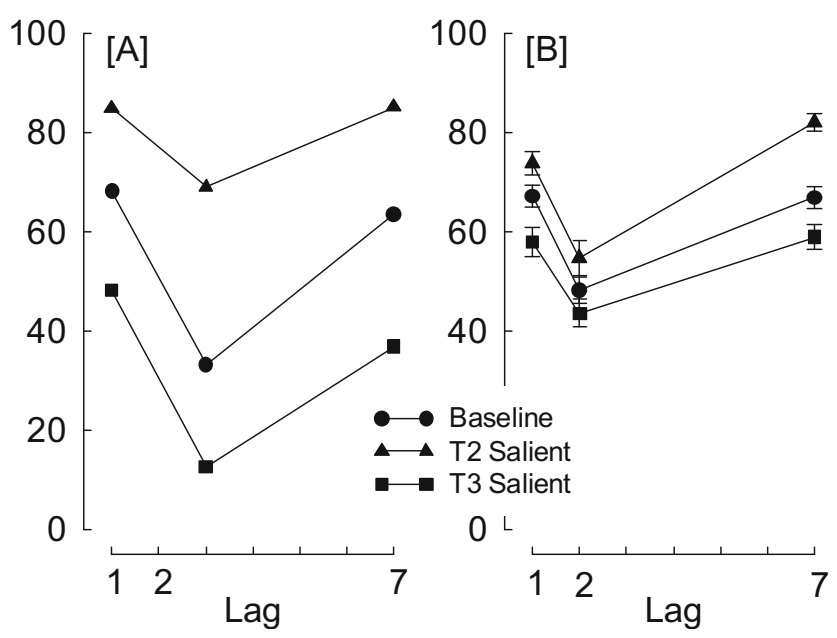

Fig. 4 (A) Simulated results of correct T2-T3 ordered responses for Experiment 2, based on the eSTST model (Wyble et al., 2009). (B) Percentage of correct T2-T3 ordered responses in Experiment 2, only for trials in which all three targets were reported correctly and T1 was reported first. Error bars indicate standard error of the mean

Procedure The design and procedures were the same as in Experiment 1, with the following exceptions. In the Baseline condition, the fixation stimulus was the \# symbol. Importantly, in the other two conditions, the fixation stimulus was a letter. Observers were instructed to attend to this letter because its identity would always be the same as one of either the $\mathrm{T} 2$ or $\mathrm{T} 3$ letters.

\section{Results and discussion}

Identification accuracy Identification accuracy (and associated standard error) for the three targets, averaged over observers, separately for each combination of Salience and Lag, is presented in Table 1. As in Experiment 1, no statistical analyses were performed, except to verify that an $A B$ was obtained. This was done by comparing the T2 means for Lags 2 and 7 in all three salience conditions. The analysis revealed significant $\mathrm{AB}$ deficits in all three conditions: Baseline: $t(34)=9.06, p<0.001$, T2 Salient: $t(34)=3.84, p$ $=0.001$; T3 Salient: $t(34)=7.56, p<0.001$.

Perception of temporal order Each of the 35 observers contributed 40 trials at each of the nine combinations of Salience (Baseline, T2 Salient, T3 Salient) and $\operatorname{Lag}(1,2,7)$, for a total of 1,400 trials per cell. The number of trials on which all three targets were reported correctly with $\mathrm{T} 1$ in the correct position was 482, 478, and 878 for Lags 1, 2, and 7, respectively, in the Baseline condition, 489, 551, and 862 for Lags 1, 2, and 7, respectively, in the T2 Salient condition, and 503, 465, and 781 in the T3 Salient condition. The functions in Fig. 4B illustrate the percentage of trials on which $\mathrm{T} 2$ and $\mathrm{T} 3$ were reported in the correct sequence, separately for each condition, across lags. A 3 (Salience: Baseline, T2 Salient, T3 Salient) ×
3 (Lag: 1,2,7) within-subjects ANOVA performed on the data in Fig. 4B yielded significant effects of Salience, $\mathrm{F}(2,68)=$ $19.28, p<0.001, \mathrm{MSE}=381.96, \eta_{\mathrm{p}}{ }^{2}=0.362$, and Lag, $\mathrm{F}(2$, $68)=57.05, p<0.001$, MSE $=224.25, \eta_{\mathrm{p}}{ }^{2}=0.627$. The interaction effect also was significant, $\mathrm{F}(4,136)=3.05, p=$ $0.019, \operatorname{MSE}=127.12, \eta_{\mathrm{p}}{ }^{2}=0.082$.

To ascertain whether the results of each of the two salience manipulations (T2 or T3 salient) differed significantly from baseline, we performed two additional planned comparisons. The T3-salient versus Baseline ANOVA revealed significant effects of Salience, $\mathrm{F}(1,34)=8.50, p=0.006, \mathrm{MSE}=331.37$, $\eta_{\mathrm{p}}{ }^{2}=0.200$, and Lag, $\mathrm{F}(2,68)=30.73, p<0.001$, MSE $=$ $214.43, \eta_{\mathrm{p}}{ }^{2}=0.475$. The interaction effect was not significant $(\mathrm{F}<1)$. The T2-salient versus Baseline ANOVA revealed significant effects of Salience, $\mathrm{F}(1,34)=26.36, p<0.001$, $\mathrm{MSE}=175.27, \eta_{\mathrm{p}}{ }^{2}=0.437$, and Lag, $\mathrm{F}(2,68)=53.67, p<$ $0.001, \mathrm{MSE}=197.04, \eta_{\mathrm{p}}{ }^{2}=0.612$. The interaction effect also was significant, $\mathrm{F}(2,68)=3.59, p=0.033, \mathrm{MSE}=119.03, \eta_{\mathrm{p}}{ }^{2}$ $=0.096$. We examined this significant interaction by performing individual $t$ tests between the mean for the Baseline condition and that for the T2-Salient condition, separately for each lag. The only comparison that did not quite reach significance was at Lag $2, t(34)=1.85, p=0.073$ (all other $p \mathrm{~s}<0.02$ ). Paralleling the finding at Lag 1 in Experiment 1 , the difference between the T2-salient and the Baseline condition, although only marginally significant, was numerically in the expected direction. In addition, the difference between the T2-salient and the T3-salient conditions at Lag 2 was found to be statistically significant $(t(34)=2.22, p=0.033)$. These observations are consistent with the idea that the salience manipulation affected the perception of temporal order at Lag 2 just as it did at other lags throughout the period of the AB.

Considered collectively, these statistical analyses confirm the graphical evidence in Fig. 4B that, relative to baseline, the perception of temporal order was more accurate when $\mathrm{T} 2$ was salient and less accurate when T3 was salient. As well as being consistent with qualitative predictions based on the principle of prior entry, this pattern of results is broadly consistent with the eSTST simulation illustrated in Fig. 4A. The differences in level between the simulated and the empirical outcomes clearly suggest the need for some adjustment in the parameters used in the simulation. For example, the $30 \%$ reduction in the tokenization threshold of salient targets is probably in need of reduction.

Combined analysis of Experiments 1 and 2 The pattern of results in Experiment 2 (Fig. 4B) parallels that seen in Experiment 1 (Fig. 3B), strongly suggesting that, in accordance with the principle of prior entry, the effect of salience on the perception of temporal order is invariant with whether salience is manipulated exogenously or endogenously. 
We explored the equivalence of the exogenous and the endogenous manipulations of salience in a separate ANOVA in which Experiment was entered as a between-subjects factor. The 3 within-subject (Salience: Baseline, T2 Salient, T3 Salient) $\times 3$ within-subject (Lag: 1, 2, 7) $\times 2$ betweensubjects (Experiment: 1, 2) mixed-factors ANOVA revealed significant effects of Salience, $\mathrm{F}(2,180)=74.29, p<0.001$, $\mathrm{MSE}=277.10 \eta_{\mathrm{p}}{ }^{2}=0.452$, and Lag, $\mathrm{F}(2,180)=145.18, p<$ $0.001, \mathrm{MSE}=234.67, \eta_{\mathrm{p}}{ }^{2}=0.617$. The Salience $\times$ Lag interaction effect was also significant, $\mathrm{F}(4,360)=5.62, p<0.001$, $\mathrm{MSE}=139.83, \eta_{\mathrm{p}}{ }^{2}=0.059$. Notably, neither the main effect of Experiment, nor any of the interactions involving Experiment were significant (all Fs $<1$ except the three-way interaction, which had $F<1.9$ ). In view of the nonsignificant effect of Experiment and its interactions, we combined the data for Experiments 1 and 2 to produce Fig. 5.

We pursued the significant interaction effect revealed in the combined ANOVA by performing individual $t$ tests among the three salience conditions, separately for each lag. All $p$ s were $<0.004$, except for the Baseline versus T2-Salient comparison at Lag $1(p=0.020)$. The uniformly significant $t$ tests in the combined analysis contrast with the finding that some of the corresponding comparisons in the individual analyses of Experiments 1 and 2 failed to reach significance. A plausible suggestion is that the failures in the individual analyses might have arisen from lack of power. In this respect, it should be noted that, even when short of significance, the numerical ordering of the means was invariably in the expected direction.

Coupled with the graphical evidence in Figs. 3B and 4B, the analysis involving both experiments confirms that the results of Experiment 2 matched those of Experiment 1 in most respects, despite the very different manipulations of salience. This correspondence is consistent with the hypothesis that the exogenous and endogenous manipulations employed in

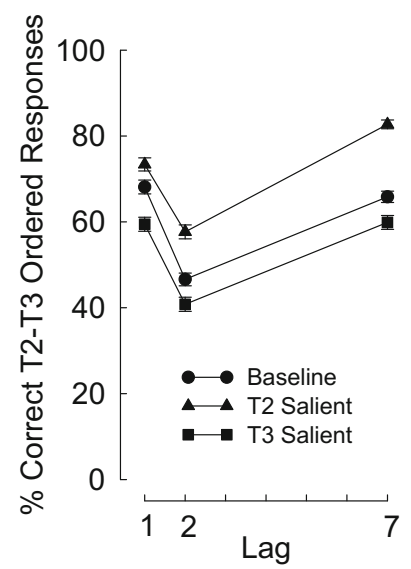

Fig. 5 Percentage of correct T2-T3 ordered responses, combined for Experiments 1 and 2, only for trials in which all three targets were reported correctly and T1 was reported first. Error bars indicate standard error of the mean
Experiments 1 and 2, operate in similar ways to affect the perception of temporal order throughout the period of the AB.

\section{General discussion}

The main objective of the present work was to examine the principle of prior entry as it applies to the perception of temporal order of two rapidly sequential targets throughout the period of the AB. The principle of prior entry specifies that perception of temporal order is affected by the relative latency at which each target is processed. In the present work, latency was manipulated by means of salience. In Experiment 1, salience was manipulated exogenously by coloring the salient target red, with all other items colored green. In Experiment 2, salience was manipulated endogenously by varying which of two targets (T2 or T3) matched the contents of working memory. The results of both experiments were quite similar to one another: salience affected the perception of temporal order throughout the period of the AB. This pattern of results was consistent with qualitative predictions based on the prior-entry principle and with formal predictions from the eSTST model.

\section{Theoretical accounts of perceived temporal order}

The prior-entry account states that the perception of temporal order of two sequential targets is determined by two factors: the actual sequence in which the targets are presented and the latency of processing. On this reasoning, the present findings are explained on the grounds that these two factors worked in conjunction with one another when the salient stimulus was T2, but in opposition when it was T3. Besides being consistent with the general principle of prior entry, the results are successfully modeled by eSTST (Figs. 3A and 4A).

An alternative account has been offered by Hommel and Akyürek (2005) in terms of episodic integration. The basic idea is that, given two targets presented in rapid sequence (T2 and T3 in the present case), the leading target opens an attentional gate that closes sluggishly, allowing the trailing target to slip through and become part of the same attentional episode. When that happens, accuracy of identification is high for both targets, but this comes at a cost: being part of the same attentional episode causes information about temporal order to be lost. As Hommel and Akyürek pointedly noted, "As subjects were able to correctly report both target identities both targets must have gained access to attentional resources. According to the sluggish-gate account this would mean that [T2 and T3] became part of the same attentional episode, which necessarily eliminated information about the sequence of the two stimuli." An implication of this account is that perception of temporal order should be at chance $(50 \%)$ whenever the targets are part of a single attentional episode. 
The finding that the percentage of order reversals at Lag 2 was at approximately $50 \%$ in the Baseline condition (Fig. 5) is in line with predictions from the episodic integration account. However, this cannot be taken as unequivocal support for that account, because the $50 \%$ level seen in the Baseline condition in Fig. 5 may have arisen not from a loss of temporal order but from a systematically skewed ordering of $\mathrm{T} 2$ and $\mathrm{T} 3$ that happened to settle at $50 \%$. This option is consistent with the present Lag-2 results for the T2-Salient and T3-Salient conditions, as well as with the results of Spalek et al. (2012) in which the percentage of order reversals decreased significantly above as well as below $50 \%$ at Lag 2 . Besides, as presently formulated, the episodic integration model cannot account for variation in order errors as a function of lag (Fig. 5). Given that (a) only trials on which both T2 and T3 were identified correctly were considered for the analysis of order reversals, and that (b) T3 was always presented directly after T2, causing the two targets to be part of the same attentional episode, the probability of order reversals should have been invariant with lag. This invariance also should be true for other manipulations, notably salience, which is not part of the episodic integration model as presently formulated.

These considerations do not necessarily invalidate the episodic integration model. The model could be expanded in several ways to encompass the present results. For example, one possibility is for two attentional episodes to be initiated in quick succession. In this case, order information would be retained, bringing the accuracy of order information above chance level. ${ }^{3}$ For such an account to be applicable to the present results, however, what needs to be articulated are the rules that govern when two stimuli become part of a single attentional episode or when they form two distinct episodes. What also is in need of explanation is how two distinct episodes can occur when the targets are presented in rapid succession (e.g., within $\sim 100 \mathrm{~ms}$ ) but not when they are separated by a longer interval $(200-500 \mathrm{~ms})$, as during the period of the $\mathrm{AB}$.

\section{Comparison with earlier work}

The influence of exogenous and endogenous factors on accuracy of $\mathrm{T} 2$ identification in the $\mathrm{AB}$ was examined by Ghorashi, Enns, Spalek, and Di Lollo (2009) and by Ghorashi, Spalek, Enns, and Di Lollo (2009) using a cueing paradigm. The critical finding was that the effect of cueingwhether exogenous or endogenous - on accuracy of T2 identification was invariant across lags. That is, the effect of cueing was additive with the overall $\mathrm{AB}$ effect. The present results reveal a similar pattern of additivity: the effect of salience on the perception of temporal order was broadly additive with the overall $\mathrm{AB}$ effect (Fig. 5).

\footnotetext{
${ }^{3}$ We thank Elkan Akyürek for this suggestion.
}

The present results are also consistent with those of Olivers, Hilkenmeier, and Scharlau (2011) who concluded that "...order reversals in the attentional blink are most parsimoniously explained through prior entry..." (p. 65). Because the manipulation of prior entry (by precuing) was implemented only at Lag 1 , which is known to be a special case characterized by relative immunity from the $\mathrm{AB}$ deficit, those results cannot be regarded as addressing the issue of prior entry throughout the period of the AB. In contrast, our three-target design permitted an assessment of the effects of prior entry throughout the period of the $\mathrm{AB}$, and provides support for the conclusion that the perception of temporal order throughout the $\mathrm{AB}$ is modulated by prior entry.

Acknowledgments This work was supported by a Canadian Foundation for Innovation New Opportunities Grant, a grant from the British Columbia Knowledge Development Fund, and a Discovery Grants from the Natural Sciences and Engineering Research Council of Canada (NSERC) to TMS, and by an Alexander Graham Bell Canada Graduate Scholarships from NSERC and a Provost Prize of Distinction from Simon Fraser University to HEPL. The authors thank Brad Wyble for helpful comments and for performing the simulations illustrated in Figs. $3 \mathrm{~A}$ and $4 \mathrm{~A}$.

\section{References}

Akyürek, E. G., \& Hommel, B. (2005). Target integration and the attentional blink. Acta Psychologica, 119, 305-314.

Akyürek, E. G., Riddell, P. M., Toffanin, P., \& Hommel, B. (2007). Adaptive control of event integration: Evidence from event-related potentials. Psychophysiology, 44, 383-391.

Akyürek, E. G., Eshuis, S. A., Nieuwenstein, M. R., Saija, J. D., Bașkent, D., \& Hommel, B. (2012). Temporal target integration underlies performance at lag 1 in the attentional blink. Journal of Experimental Psychology: Human Perception and Performance, $38,1448-1464$.

Chun, M. M. (1997). Temporal binding errors are redistributed in the attentional blink. Perception \& Psychophysics, 59, 1191-1199.

Chun, M. M., \& Potter, M. C. (1995). A two-stage model for multiple target detection in rapid serial visual presentation. Journal of Experimental Psychology: Human Perception and Performance, 21, 109-127.

Donk, M., \& Soesman, L. (2011). Object salience is transiently represented whereas object presence is not: Evidence from temporal order judgment. Perception, 40, 63-73.

Downing, P. E. (2000). Interactions between visual working memory and selective attention. Psychological Science, 11, 467-473.

Fecteau, J. H., \& Munoz, D. P. (2006). Salience, relevance, and firing: A priority map for target selection. Trends in Cognitive Sciences, 10, 382-390.

Fortier-Gauthier, U., Dell'Acqua, R., \& Jolicœur, P. (2013). The "redalert" effect in visual search: Evidence from human electrophysiology. Psychophysiology, 50, 671-679.

Ghorashi, S., Enns, J. T., Spalek, T. M., \& Di Lollo, V. (2009a). Spatial cuing does not affect the magnitude of the attentional blink. Attention, Perception, \& Psychophysics, 71, 989-993.

Ghorashi, S., Spalek, T. M., Enns, J. T., \& Di Lollo, V. (2009b). Are spatial selection and identity extraction separable when attention is controlled endogenously? Attention, Perception, \& Psychophysics, $71,1233-1240$. 
Hilkenmeier, F., Scharlau, I., Weiss, K., \& Olivers, C. N. L. (2012). The dynamics of prior entry in serial visual processing. Visual Cognition, 20, 48-76.

Hommel, B., \& Akyürek, E. G. (2005). Lag-1 sparing in the attentional blink: Benefits and costs of integrating two events into a single episode. The Quarterly Journal of Experimental Psychology, 58A, $1415-1433$

Isaak, M. I., Shapiro, K. L., \& Martin, J. (1999). The attentional blink reflects retrieval competition among multiple rapid serial visual presentation items: Tests of an interference model. Journal of Experimental Psychology: Human Perception and Performance, $25,1774-1792$

Jaskowski, P. (1993). Selective attention and temporal-order judgment. Perception \& Psychophysics, 22, 681-689.

Kanwisher, N. (1987). Repetition blindness: Type recognition without token individuation. Cognition, 27, 117-143.

Lagroix, H. E. P., Spalek, T. M., Wyble, B., Jannati, A., \& Di Lollo, V. (2012). The root cause of the attentional blink: First-target processing or disruption of input control? Attention, Perception, \& Psychophysics, 74, 1606-1622.

Maylor, E. A., \& Hockey, R. (1985). Inhibitory component of externally controlled covert orienting in visual space. Journal of Experimental Psychology: Human Perception and Performance, 11, 777-787.

Olivers, C. N., \& Meeter, M. (2008). A boost and bounce theory of temporal attention. Psychological Review, 115, 836.

Olivers, C. N. L., Hilkenmeier, F., \& Scharlau, I. (2011). Prior entry explains order reversals in the attentional blink. Attention, Perception, \& Psychophysics, 73, 53-67.

Raymond, J. E., Shapiro, K. L., \& Arnell, K. M. (1992). Temporary suppression of visual processing in an RSVP task: An attentional blink? Journal of Experimental Psychology: Human Perception and Performance, 18, 849-860.

Reeves, A., \& Sperling, G. (1986). Attention gating in short-term visual memory. Psychological Review, 93, 180-206.

Shih, S. I. (2008). The attention cascade model and attentional blink. Cognitive Psychology, 56, 210-236.

Shore, D. I., Spence, C., \& Klein, R. M. (2001). Visual prior entry. Psychological Science, 12, 205-212.
Soto, D., Heinke, D., Humphreys, G. W., \& Blanco, M. J. (2005). Early, involuntary top-down guidance of attention from working memory. Journal of Experimental Psychology: Human Perception and Performance, 31, 248-261.

Spalek, T. M., Falcon, L. J., \& Di Lollo, V. (2006). Attentional blink and attentional capture: Endogenous versus exogenous control over paying attention to two important events in close succession. Perception \& Psychophysics, 68, 674-684.

Spalek, T. M., Lagroix, H. E. P., Yanko, M. R., \& Di Lollo, V. (2012). Perception of temporal order is impaired during the time course of the attentional blink. Journal of Experimental Psychology: Human Perception and Performance, 38, 402-413.

Spence, C., \& Parise, C. (2010). Prior-entry: A review. Consciousness and Cognition, 19, 364-379.

Spence, C., Shore, D. I., \& Klein, R. M. (2001). Multisensory prior entry. Journal of Experimental Psychology: General, 130, 799-832.

Stelmach, L. B., \& Herdman, C. M. (1991). Directed attention and perception of temporal order. Journal of Experimental Psychology: Human Perception and Performance, 17, 539-550.

Theeuwes, J. (1992). Perceptual selectivity for color and form. Perception \& Psychophysics, 51, 599-606.

Titchener, E. B. (1908). Lectures on the elementary psychology of feeling and attention. New York: Macmillan.

Töllner, T., Zehetleitner, M., Gramann, K., \& Müller, H. J. (2011). Stimulus saliency modulates pre-attentive processing speed in human visual cortex. Plos One, 6, 1-8.

Tünnermann, J., Petersen, A., \& Scharlau, I. (2015). Does attention speed up processing? Decreases and increases in processing rate in visual prior entry. Journal of Vision, 15, 1-27.

Weiss, K., Hilkenmeier, F., \& Scharlau, I. (2013). Attention and the speed of information processing: Posterior entry for unattended stimuli instead of prior entry for attended stimuli. PLoS ONE, 8, 1-8.

Wyble, B., Bowman, H., \& Nieuwenstein, M. (2009). The attentional blink provides episodic distinctiveness: Sparing at a cost. Journal of Experimental Psychology: Human Perception and Performance, $35,787-807$. 